\title{
A ERGONOMIA COMO FATOR ECONÔMICO E COMPETITIVO PARA PEQUENAS EMPRESAS
}

\section{ERGONOMICS AS AN ECONOMIC AND COMPETITIVE FACTOR REGARDING SMALL COMPANIES}

\author{
Thiago de Oliveira Pegatin ${ }^{1}$; Antonio Augusto de Paula Xavier ${ }^{2 ;}$ Luiz Alberto Pilatti ${ }^{3}$; \\ Ariel Orlei Michaloski ${ }^{4}$ \\ ${ }^{1}$ BioProdução - UTFPR - Ponta Grossa - Brasil - thiagopegatin@yahoo.com.br \\ ${ }^{2}$ BioProdução - UTFPR - Ponta Grossa - Brasil - augustox@utfpr.edu.br \\ ${ }^{3}$ BioProdução - UTFPR - Ponta Grossa - Brasil - lapilatti@utfpr.edu.br \\ ${ }^{4}$ UTFPR - Ponta Grossa - Brasil - ariel@interponta.com.br
}

\begin{abstract}
Resumo
O estudo relata os resultados de um programa de ergonomia em uma pequena indústria metalúrgica, responsável pela fabricação de autopeças para indústria automobilística. Teve como objetivo caracterizar a relação de custo/benefício do enfoque preventivo, bem como o impacto econômico gerado com sua implementação. Para o estudo das situações de trabalho foi utilizada a metodologia de Análise Ergonômica do Trabalho. O projeto de intervenção compreendeu adequações ergonômicas, atividades educacionais e um programa de exercícios laborais, adequados segundo a metodologia $5 \mathrm{~W} 2 \mathrm{H}$. Foram quantificados ainda os índices de horas perdidas com absenteísmo por meio do cartão de ponto dos funcionários pré e pós-implementação do programa. Os resultados demonstram um aumento de $9,74 \%$ em horas trabalhadas e uma redução de 49,43\% no índice de absenteísmo na comparação entre os periodos pré e pós-pesquisa. Apontou ainda um tempo de retorno do investimento de oito meses, com um saldo de \$28.830,89 dólares ao final do estudo. Concluiu-se que um programa preventivo estruturado segundo as bases metodológicas da ergonomia e da engenharia econômica pode atuar como fator competitivo $e$ econômico nas empresas.
\end{abstract}

Palavras-chave: Ergonomia, Engenharia Econômica, Pequenas empresas.

\section{Introdução}

Ao longo das ultimas décadas, as pequenas e médias empresas têm enfrentado diversas modificações associadas ao processo de inovação tecnológica e de reestruturação produtiva e organizacional.

Desta forma, a interdisciplinaridade se faz cada vez mais presente e necessária, em todos os níveis hierárquicos das organizações.

Como ciência que busca compreender o trabalho para transformá-lo a ergonomia vem sendo cada vez mais solicitada para garantir a melhoria das condições de saúde, segurança, das relações 
interpessoais e para contribuir com o sucesso técnico, econômico e financeiro das novas tecnologias (GUERIN et al, 2001; WISNER, 2004).

No entanto, os pilares conceituais da ergonomia ainda carecem de difusão à outras áreas de conhecimento, principalmente àquelas constituídas por processos extremamente técnicos, como por exemplo na quantificação do fator econômico e competitivo nas empresas.

Para que os resultados obtidos com aplicação dos programas de ergonomia emirjam com maior aplicabilidade ao setor empresarial, se faz necessário a aplicação de conceitos advindos da engenharia econômica como forma de apoio à decisão por parte dos empresários.

A engenharia econômica aplica seus conhecimentos a alternativas provindas de fatores técnicos, financeiros e sociais, procurando encontrar aquela que forneça a otimização dos recursos (HIRSCHFELD, 1986). Em complemento, Casarotto Filho \& Kopittke (1998) relatam que a engenharia econômica objetiva a análise econômica de decisões sobre investimentos.

Inserida neste contexto de alta competitividade, a gestão de recursos das micro e pequenas empresas merece especial atenção.

Em um estudo de caso com microempresas do setor moveleiro, Nunes \& Franzoni (2004) verificaram que a gestão do processo aliada a ergonomia promove um ambiente favorável dentro das organizações, capaz de manter a eficiência do processo e a qualidade da produção.

Alberton et. al. (2004) defendem que em um ambiente altamente competitivo e de recursos limitados, a aplicação adequada dos recursos de uma empresa proporciona não apenas o aumento de seu valor, mas também é fator decisivo para sua sobrevivência.

A área de saúde e segurança do trabalho, no entanto, ainda parece distante das micro e pequenas empresas quando se trata de análise de investimentos.

As ações em saúde e segurança do trabalho, incluindo-se as ações ergonômicas, restringemse em sua grande maioria, por situações de coerção, em que a fiscalização do Ministério do Trabalho obriga, mediante notificação oficial, o ajuste das condições de trabalho.

No entanto, Barkokébas Júnior et. al. (2006) ressaltam que se deve ter em mente que promover a segurança do trabalho é economicamente vantajosa; além da obrigação legal, é dever moral, devido aos aspectos sociais envolvidos, causando danos a todos os segmentos: empresas, trabalhadores e sociedade; resultando para todos, custo econômico e humano.

Para tanto, o presente estudo se pretende a responder o seguinte questionamento: Como um programa de ergonomia pode fazer parte da análise de investimentos de micro e pequenas empresas? 
Supõe-se que um programa de ergonomia estruturado segundo uma abordagem participativa em vinculação com os conceitos advindos da engenharia econômica, como tentativa na quantificação do valor da prestação de serviço no tempo, pode auxiliar as micro e pequenas empresas a estruturarem sua condição organizacional e inserir estes programas em sua análise de investimentos.

Esta pesquisa objetivou, portanto, quantificar a relação custo/benefício na implementação de um programa ergonômico em uma pequena empresa, bem como caracterizar as ações metodológicas necessárias para o alcance proposto.

\section{Metodologia}

\subsection{População e amostra}

A pesquisa foi realizada em uma pequena empresa metalúrgica, responsável pela produção de induzidos para motor de partida, estatores e rotores utilizados na indústria automobilística, situada na região centro-oeste paulista, e compreendeu o período de março de 2005 a março de 2006.

A empresa conta com 35 funcionários em seu quadro funcional, sendo que $31(88,6 \%)$ são do sexo masculino e $4(11,4 \%)$ do sexo feminino, com média de idade de 24,8 $( \pm 8,58)$ anos. Em relação ao nível de escolaridade, nota-se que 07 (20,0\%) funcionários possuem o ensino fundamental completo, $19(54,3 \%)$ o ensino médio, 06 (17,1\%) o ensino técnico profissionalizante e $3(8,57 \%)$ possuem ou estão cursando o ensino superior.

\subsection{Instrumentos de coleta e tratamentos dos dados}

A primeira etapa da pesquisa compreendeu o levantamento de dados referentes ao cartão de ponto dos funcionários, no período de março de 2004 a fevereiro de 2006 para quantificação dos custos gerados pelo índice de absenteísmo, representados por faltas justificadas ou não.

Para análise dos dados foi utilizada estatística descritiva comparativa, levando em consideração a quantidade de horas perdidas com o absenteísmo em relação às horas trabalhadas no mês.

Foram ainda analisados e comparados, o valor monetário das perdas em relação ao valor investido no programa ergonômico, com intuito de apontar o tempo de retorno e de compensação financeira do programa. 
Os indicadores utilizados foram dólares (\$), e estipulados pela empresa em conjunto com uma consultoria, compreendendo o custo gerado pelo absenteísmo e o valor total do investimento no programa preventivo.

\subsection{Procedimentos técnicos}

Para o estudo das situações trabalho foi utilizado a metodologia de Análise Ergonômica do Trabalho (AET) e compreendeu basicamente as etapas propostas por Guérin et al. (2001):

- Análise da demanda: onde foi realizado o trabalho de análise e reformulação da demanda, apresentando uma proposta de ação, os meios necessários e os prazos de execução;

- Análise da tarefa: análise das condições determinadas pela empresa aos trabalhadores;

- Análise da atividade: observação das atividades, fotos, filmagens e verbalizações, com objetivo de identificar riscos físicos, cognitivos e organizacionais no trabalho;

- Caderno de encargos: caracterizou-se pelo projeto de intervenção ergonômica.

O projeto de intervenção compreendeu três etapas: adequações ergonômicas (1), programas educacionais (2) e um programa de exercícios laborais (3), adequados segundo a técnica 5W2H (STRAUSS \& CORBIN, 1990), apresentada no quadro 1.

Quadro 1 - Projeto de intervenção segundo metodologia 5W2H

\begin{tabular}{|c|c|c|c|c|c|c|}
\hline Ação & What? & Who? & When? & Where? & Why? & How? \\
\hline 1 & $\begin{array}{l}\text { Adequação de } \\
\text { posto de } \\
\text { trabalho }\end{array}$ & $\begin{array}{c}\text { Direção da } \\
\text { empresa }+ \\
\text { fisioterapeuta }\end{array}$ & Abril de 2005 & $\begin{array}{c}\text { Setor } \\
\text { administrativo }\end{array}$ & $\begin{array}{c}\text { Inadequações físicas e } \\
\text { organizacionais }\end{array}$ & $\begin{array}{c}\text { Ajuste dos } \\
\text { monitores, cadeiras e } \\
\text { equipamentos }\end{array}$ \\
\hline 1 & $\begin{array}{l}\text { Adequação de } \\
\text { posto de } \\
\text { trabalho }\end{array}$ & $\begin{array}{c}\text { Direção da } \\
\text { empresa }+ \\
\text { fisioterapeuta }\end{array}$ & Abril de 2005 & $\begin{array}{l}\text { Setor de } \\
\text { produção }\end{array}$ & $\begin{array}{c}\text { Inadequações físicas e } \\
\text { organizacionais }\end{array}$ & $\begin{array}{l}\text { Ajuste de altura da } \\
\text { bancada de trabalho } \\
\text { e disposição dos } \\
\text { equipamentos }\end{array}$ \\
\hline 2 & $\begin{array}{l}\text { Apresentação } \\
\text { do programa }\end{array}$ & Fisioterapeuta & $\begin{array}{l}\text { Março de } \\
2005\end{array}$ & $\begin{array}{l}\text { Todos os } \\
\text { setores }\end{array}$ & $\begin{array}{l}\text { Sensibilização: } \\
\text { importância do } \\
\text { programa }\end{array}$ & Palestra \\
\hline 2 & $\begin{array}{l}\text { Orientações } \\
\text { ergonômico- } \\
\text { operacionais }\end{array}$ & Fisioterapeuta & $\begin{array}{c}\text { Primeira } \\
\text { segunda-feira } \\
\text { de cada mês }\end{array}$ & $\begin{array}{l}\text { Todos os } \\
\text { setores }\end{array}$ & $\begin{array}{l}\text { Discussão de temas } \\
\text { inerentes a cada setor }\end{array}$ & $\begin{array}{c}\text { Palestras focais para } \\
\text { cada setor em } \\
\text { específico }\end{array}$ \\
\hline 2 & $\begin{array}{l}\text { Diálogos } \\
\text { ergonômicos }\end{array}$ & $\begin{array}{l}\text { Fisioterapeuta } \\
+ \text { Educador } \\
\text { físico }\end{array}$ & $\begin{array}{l}\text { Diálogos a } \\
\text { cada } 15 \text { dias }\end{array}$ & $\begin{array}{l}\text { Todos os } \\
\text { setores }\end{array}$ & $\begin{array}{l}\text { Discussão de temas } \\
\text { gerais em saúde e } \\
\text { segurança }\end{array}$ & $\begin{array}{l}\text { Mini-palestras } \\
\text { durante o período da } \\
\text { ginástica }\end{array}$ \\
\hline
\end{tabular}




\begin{tabular}{|c|c|c|c|c|c|c|}
\hline 3 & $\begin{array}{c}\text { Programa de } \\
\text { exercícios } \\
\text { laborais }\end{array}$ & $\begin{array}{c}\text { Fisioterapeuta } \\
+ \text { Educador } \\
\text { físico }\end{array}$ & $\begin{array}{c}\text { Segunda, } \\
\text { quarta e } \\
\text { sexta-feira }\end{array}$ & $\begin{array}{c}\text { Pátio da } \\
\text { empresa (todos } \\
\text { os setores) }\end{array}$ & $\begin{array}{c}\text { Incentivo à atividade } \\
\text { física, melhora da } \\
\text { condição corporal }\end{array}$ & $\begin{array}{c}3 \text { vezes na semana, } \\
\text { antes do trabalho, } \\
\text { com duração de 15 } \\
\text { minutos }\end{array}$ \\
\hline
\end{tabular}

Fonte: Pesquisa realizada pelos autores

Como a empresa não apresentou afastamentos por doenças ocupacionais no período destinado ao estudo, o custo de absenteísmo foi estipulado pelo valor monetário gerado pela hora trabalhada do funcionário com faltas justificadas (atestados) ou não, mais o valor produtivo esperado por hora de trabalho do mesmo funcionário.

O investimento do programa (indicador How much?) incluiu as horas de trabalho de uma consultoria que prestou serviço à empresa, compreendendo um investimento mensal de 12 horas de consultoria. Os valores foram estipulados em dólares (\$) e são apresentados na tabela 1.

Tabela 1 - Valores de referência empregados no estudo (How much?)

\begin{tabular}{cccc}
\hline Indicador & Valor unitário (\$) & Mês & Ano \\
\hline Custo absenteísmo & 19,50 & UM x Número de horas perdidas \\
Investimento & 24,40 & 12 horas & 144 horas \\
\hline
\end{tabular}

Fonte: Pesquisa realizada pelos autores

\section{Resultados}

A demanda pelo estudo se deu pelo fato de que a empresa em questão apresentava ao início de 2005, um plano de expansão que, pretendia elevar até o final de 2005 em 5\% sua capacidade produtiva, mantendo o mesmo quadro de funcionários.

A pesquisa ateve-se ao índice de absenteísmo, gerado por faltas justificadas ou não, como possível fator de desempenho no auxílio à meta pré-estabelecida.

O levantamento de horas trabalhadas no período anterior ao estudo, de março de 2004 a fevereiro de 2005 apontou uma média de 4.186: 00:15 horas/mês, e de 4637:30:00 horas/mês no período compreendido pela pesquisa, de março de 2005 a fevereiro de 2006. A distribuição de horas trabalhadas é representada na figura 1. 
Figura 1 - Distribuição mensal de horas trabalhadas

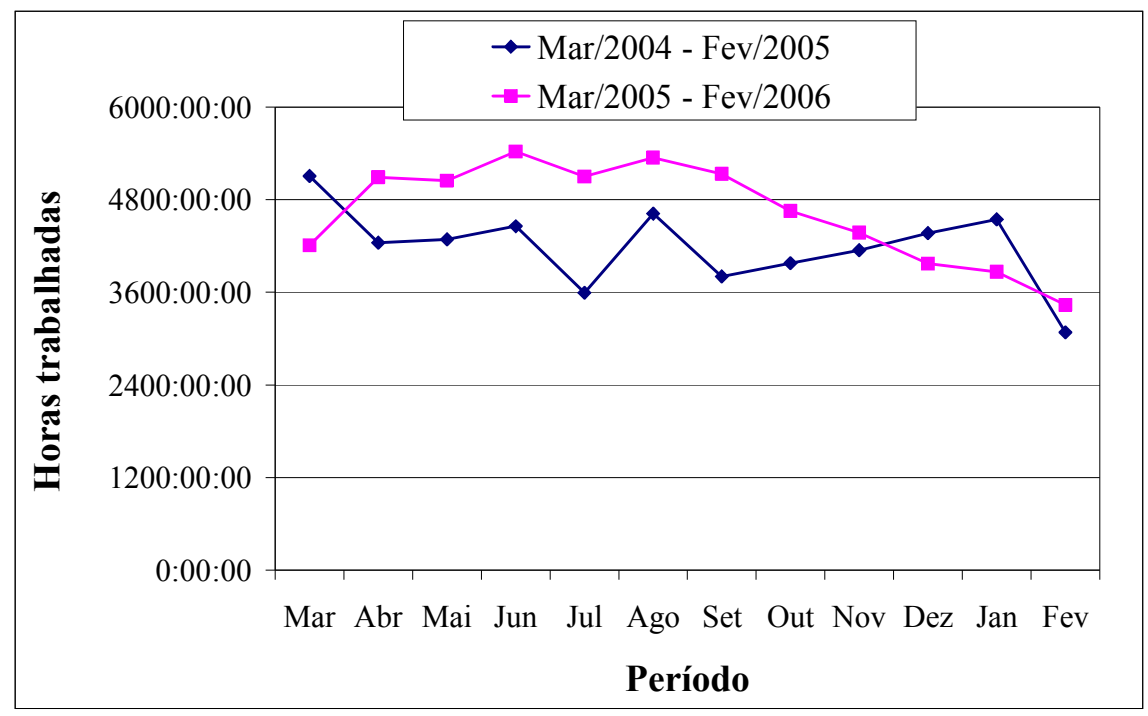

Fonte: Pesquisa realizada pelos autores

Nota-se que no primeiro mês da pesquisa, em março de 2005, onde ações efetivas ainda não estavam implementadas, não houve inversão de valores na quantidade de horas trabalhadas. Porém, a partir de abril observa-se uma inversão na curva.

Pode-se observar, na figura 2, que as perdas em horas ocasionadas pelo absenteísmo seguem inversão similar, de modo que março foi o único mês em que se contabilizou mais em perdas em relação ao mesmo período do ano anterior.

Figura 2 - Evolução de perdas mensais em horas

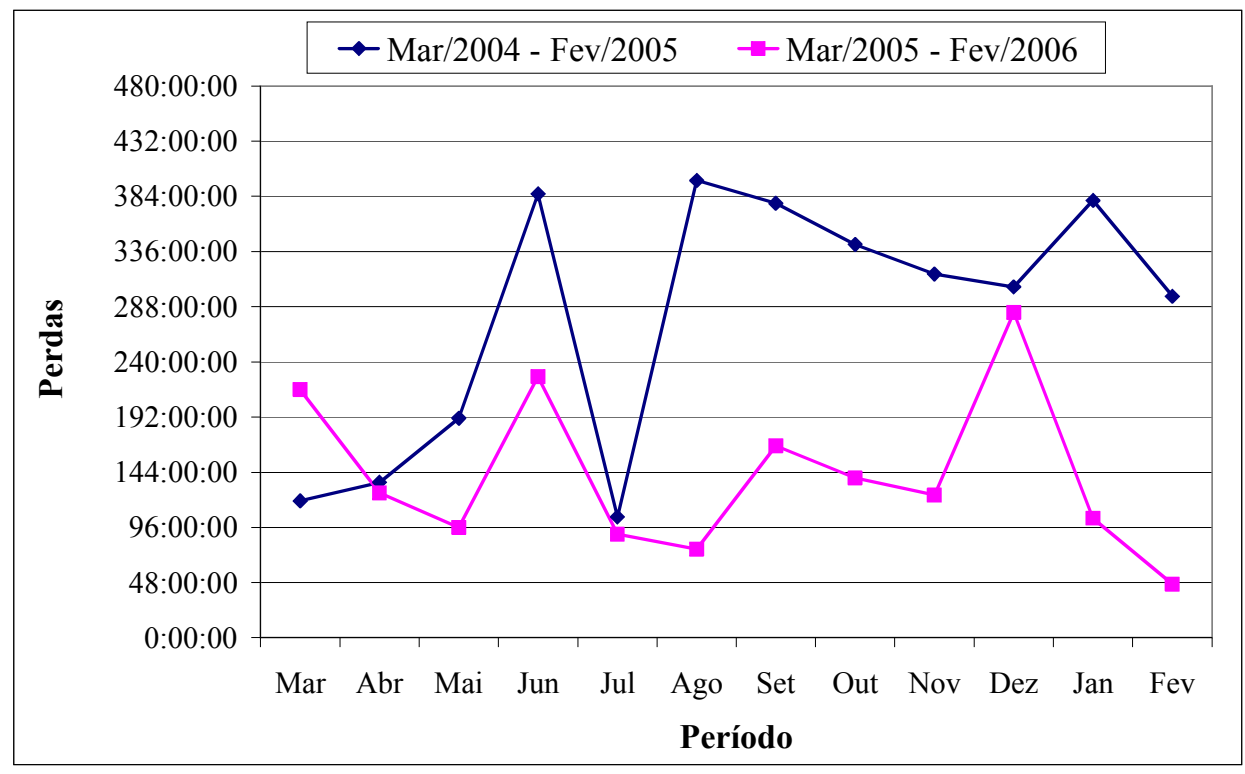

Fonte: Pesquisa realizada pelos autores

Para efeito de comparação, foram confrontados os índices percentuais de absenteísmo entre 
os períodos pré e pós-implantação do programa. O período anterior à pesquisa apresentou uma média mensal de 6,78\% $( \pm 0,03)$ em horas perdidas pelo absenteísmo. Este valor foi reduzido a uma média mensal de $3,11 \%( \pm 0,02)$ no período compreendido pelo estudo.

A distribuição do índice de absenteísmo, aponta ao final do estudo, uma redução de 1.657:36:00 horas perdidas, e que, no período do estudo, apenas os meses de março e dezembro apresentaram uma relação percentual superior $(3,06 \%$ e $0,14 \%$ respectivamente, com destaque em vermelho) em relação ao mesmo período do ano anterior (Tabela 2).

Tabela 1 - Distribuição de perdas por absenteísmo, em horas (\%), antes e após desenvolvimento do estudo

\begin{tabular}{|c|c|c|c|c|c|c|c|c|}
\hline \multicolumn{9}{|c|}{ Índice de absenteísmo } \\
\hline \multirow[b]{2}{*}{ Mês } & \multicolumn{3}{|c|}{ Antes } & \multicolumn{3}{|c|}{ Depois } & \multicolumn{2}{|c|}{ Comparativo } \\
\hline & Ano & Horas & $(\%)$ & Ano & Horas & $(\%)$ & Horas & $(\%)$ \\
\hline Março & 2004 & 119:01:00 & $(2,33)$ & 2005 & 216:00:00 & $(5,39)$ & $96: 59: 00$ & $(3,06)$ \\
\hline Abril & 2004 & $135: 13: 00$ & $(3,19)$ & 2005 & 126:00:00 & $(2,48)$ & 9:13:00 & $(0,71)$ \\
\hline Maio & 2004 & 191:00:00 & $(4,45)$ & 2005 & 96:00:00 & $(1,90)$ & 95:00:00 & $(2,55)$ \\
\hline Junho & 2004 & 386:03:00 & $(8,66)$ & 2005 & $227: 12: 00$ & $(4,19)$ & $158: 51: 00$ & $(4,47)$ \\
\hline Julho & 2004 & 105:00:00 & $(2,92)$ & 2005 & 90:00:00 & $(1,76)$ & $15: 00: 00$ & $(1,16)$ \\
\hline Agosto & 2004 & 397:49:00 & $(8,61)$ & 2005 & 77:00:00 & $(1,44)$ & $320: 49: 00$ & $(7,17)$ \\
\hline Setembro & 2004 & 378:00:00 & $(9,93)$ & 2005 & 167:00:00 & $(3,25)$ & 211:00:00 & $(6,68)$ \\
\hline Outubro & 2004 & 342:07:00 & $(8,60)$ & 2005 & 139:00:00 & $(2,99)$ & 203:07:00 & $(5,62)$ \\
\hline Novembro & 2004 & $316: 26: 00$ & $(7,63)$ & 2005 & 124:00:00 & $(2,84)$ & $192: 26: 00$ & $(4,80)$ \\
\hline Dezembro & 2004 & 305:09:00 & $(6,99)$ & 2005 & 283:00:00 & $(7,13)$ & 22:09:00 & $(0,14)$ \\
\hline Janeiro & 2005 & 380:30:00 & $(8,37)$ & 2006 & 104:00:00 & $(2,65)$ & 276:30:00 & $(5,72)$ \\
\hline Fevereiro & 2005 & 297:00:00 & $(9,64)$ & 2006 & $46: 30: 00$ & $(1,34)$ & 250:30:00 & $(8,29)$ \\
\hline Cumulativo & & $3.353: 18: 00$ & & & $1.695: 42: 00$ & & $1.657: 36: 00$ & \\
\hline Média & & $279: 26: 30$ & $(6,78)$ & & 141:18:30 & $(3,11)$ & & \\
\hline
\end{tabular}

Para análise do investimento foi realizada uma verificação entre o custo das perdas por absenteísmo e o valor total do investimento do programa, segundo os parâmetros apresentados na tabela 1 .

O valor total do investimento foi de $\$ 3.512,20$, sendo distribuídos ao longo de doze meses compreendidos pelo estudo (\$292,68 mensais). Este valor envolveu os custos com consultoria e adequações no ambiente de trabalho.

O valor total da perda com absenteísmo atingiu, no período anterior ao programa, $\$$ $65.429,91$ e, $\$ 36.599,02$ no período de estudo. O saldo entre os dois períodos foi de $\$ 28.830,89$, sendo que o tempo de retorno do investimento no programa foi de oito meses (destaque em azul).

A tabela 3 mostra a distribuição mensal de perdas com absenteísmo antes do programa em relação ao total de perdas durante o estudo. 
Tabela 2 - Distribuição de perdas por absenteísmo

\begin{tabular}{|c|c|c|c|c|c|c|}
\hline \multicolumn{7}{|c|}{ Custo do absenteísmo } \\
\hline \multirow{2}{*}{ Período } & \multicolumn{3}{|c|}{ Antes } & \multicolumn{3}{|c|}{ Depois } \\
\hline & Ano & Perda (\$) & Cumulativo & Ano & Perda + Investimento & Cumulativo \\
\hline Março & \multirow{10}{*}{2004} & $2.322,28$ & $2.322,28$ & \multirow{10}{*}{2005} & $4.507,32$ & $4.507,32$ \\
\hline Abril & & $2.638,05$ & $4.960,32$ & & $2.751,22$ & $7.258,54$ \\
\hline Maio & & $3.726,83$ & $8.687,15$ & & $2.167,85$ & $9.424,39$ \\
\hline Junho & & $7.532,68$ & $16.219,84$ & & $4.725,85$ & $14.150,24$ \\
\hline Julho & & $2.048,78$ & $18.268,62$ & & $2.048,78$ & $16.199,02$ \\
\hline Agosto & & $7.762,27$ & $26.030,89$ & & $1.795,12$ & $17.994,15$ \\
\hline Setembro & & $7.375,61$ & $33.406,50$ & & $3.551,22$ & $21.545,37$ \\
\hline Outubro & & $6.675,45$ & $40.081,95$ & & $3.004,88$ & $24.550,24$ \\
\hline Novembro & & $6.174,31$ & $46.256,26$ & & $2.712,20$ & $27.262,44$ \\
\hline Dezembro $\mathcal{J}$ & & $5.954,15$ & $52.210,40$ & & $5.814,63$ & $33.077,07$ \\
\hline Janeiro & \multirow{2}{*}{2005} & $7.424,39$ & $59.634,79$ & \multirow{2}{*}{2006} & $2.321,95$ & $35.399,02$ \\
\hline Fevereiro & & $5.795,12$ & $65.429,91$ & & $1.200,00$ & $36.599,02$ \\
\hline Média anual & & $5.452,52$ & & & $3.445,53$ & \\
\hline
\end{tabular}

O cruzamento entre os dados apresentados nas tabelas 2 e 3 revela um aumento de $9,74 \%$ no total de horas trabalhadas e de 49,43\% na redução de horas perdidas (Tabela 3).

Tabela 3 - Número de horas trabalhadas x Absenteísmo

\begin{tabular}{cccc}
\hline & Mar/04 - Fev/05 & Mar/05 - Fev/06 & Escore \\
\hline Horas trabalhadas & $50.232: 03: 00$ & $55650: 00: 00$ & Aumento de $9,74 \%$ \\
Absenteísmo & $3353: 18: 00$ & $1695: 42: 00$ & Redução de $49,43 \%$ \\
\hline
\end{tabular}

Fonte: Pesquisa realizada pelos autores

\section{Discussão}

O trabalho teve como proposta o desenvolvimento de um programa de ergonomia em uma pequena empresa, com foco às questões econômicas obtidas com o enfoque preventivo.

Observou-se mediante o estudo que a ergonomia como fator econômico e competitivo pode ser utilizado como método para análise de investimentos, inclusive nas pequenas empresas.

Em pesquisa junto aos microempresários do ramo do comércio varejista de confecções, em relação ao sistema de fluxo de caixa, Kaspczak e Sandelari (2004) observaram que existem falhas na administração financeira das micro e pequenas empresas. Apontaram ainda que a administração não possui ferramentas capazes de fornecer subsídios para um maior controle de seus recursos financeiros cotidianos, relevantes para um bom desempenho no mercado altamente competitivo.

Segundo Souza \& Clemente (1995) o grande campo de aplicação das técnicas de análise de investimentos ainda está associado ao processo de geração de indicadores utilizados na seleção de 
alternativas de investimentos.

De acordo com os resultados observados pelo estudo, supõe-se que a ergonomia pode fazer parte desta análise de investimentos no contexto empresarial.

A concepção do valor do dinheiro no tempo funciona com premissa básica nos fundamentos da engenharia econômica (FREITAS, 2002) e segundo Gitman (1997) está relacionada com uma visão de longo prazo, na qual a continuidade da empresa e seu valor devem ser observados sob a ótica de fluxos de entradas e saídas.

A ergonomia como ciência que estuda as inter-relações entre as características técnicas, humanas e organizacionais presentes no ambiente de trabalho, tem seu foco na atividade de trabalho.

Para tanto, esta pesquisa busca o centro de estudo na relação de entradas e saídas humanas, aqui representadas pelo índice de absenteísmo, quantificados pelas faltas ao trabalho, justificadas ou não.

Estudos como os de Poosanthanasarn et al. (2005) e de Carrivick et al (2005) apontam que intervenções ergonômicas reduzem as despesas médicas com tratamentos por doenças ocasionadas no trabalho, sendo que Carrivick et al (2005) relatam ainda que o programa foi capaz de reduzir em $35 \%$ as horas perdidas com tais lesões.

Porém, Bos et al (2006) apontam fortes evidencias entre as intervenções ocupacionais com a redução do desconforto físico e melhora no desempenho técnico das atividades de levantamento e transporte, porém, insuficientes para redução do absenteísmo.

Este estudo pretendeu avançar um pouco mais nesta questão, quantificando não somente o percentual de horas perdidas, mais também a relação custo-benefício do enfoque preventivo.

Observou-se que a redução de horas perdidas com absenteísmo com um ano de implantação do programa foi responsável por um retorno de U\$ 28.830,89 dólares quando comparados os períodos pré e pós-pesquisa. Foi notado ainda que o investimento no programa tem um retorno, neste caso, com oito meses após o início das atividades, atingindo um índice de aumento em horas trabalhadas de $9,74 \%$ em relação ao ano anterior.

Este dado merece especial atenção pelo fato que o número de funcionários manteve-se o mesmo durante todo o período do estudo, apresentando inclusive, diminuição de dois funcionários ao início de 2006.

Porém, verificou-se por meio da distribuição de horas perdidas apresentadas na figura 2, que o mês de dezembro apresentou um pico fora do padrão de redução que se seguiu ao longo do 
estudo.

Em análise junto à empresa, ficou constatado que os índices de absenteísmo revelados no cartão de ponto em dezembro de 2005 não se relacionavam à custos ocupacionais, e sim a duas cirurgias de estética realizadas por duas profissionais do setor administrativo.

De fato, os ganhos para empresa como fator intrínseco aos resultados de uma intervenção ergonômica, são importantes quando quantificados para fortalecimento da aplicação metodológica destas ações, assim como para fazer parte das análises de investimentos no meio empresarial.

\title{
5. Conclusão
}

Concluiu-se, ao final deste estudo, que um programa preventivo estruturado segundo as bases metodológicas da ergonomia e da engenharia econômica pode atuar como fator competitivo e econômico nas empresas.

Pode ainda auxiliar as pequenas empresas brasileiras a transpor barreiras financeiras enfrentadas pelo atual cenário competitivo e otimizar seus sistemas produtivos de modo a permear sua continuidade e oferecer meios de auxilio à tomada de decisões.

O trabalho teve ainda o anseio de referendar futuros estudos no campo da ergonomia, com intuito de fornecer uma ferramenta metodológica para quantificação do retorno financeiro gerado pelo enfoque preventivo.

Recomenda-se, no entanto, que os programas sejam planejados segundo métodos e técnicas de gestão e que envolvam a participação de toda organização. As atribuições devem ser claras e objetivas, de forma que todos realmente possam estar comprometidos com os objetivos propostos.

\begin{abstract}
The study reports the results of an ergonomics program in a small metallurgical industry, responsible for the manufacture of auto to auto industry. We aimed to characterize the cost-benefit approach to preventive as well as the economic impact generated with its implementation. To study the situations of work was used the methodology of Ergonomics Analysis of Labor. The intervention project comprised the ergonomic adjustments, educational activities and an exercise program, according to the methodology $5 \mathrm{~W} 2 \mathrm{H}$. We also quantified the rates of hours lost to absenteeism through the card section of officials before and after the program implementation. The results show an increase of $9,74 \%$ in hours worked and a reduction of $49,43 \%$ in the rate of absenteeism in the comparison between the periods before and after the search. It has also pointed out a long return on investment of eight months, with a balance of U\$ 28830,89 dollars at the end of the study. It was concluded that a preventive program structured according to the methodological foundations of ergonomics and economic engineering can act as an economic and competitive factor regarding small companies.
\end{abstract}


Key-words: Ergonomics, Economic Engineering, Small companies.

\section{Referências}

ALBERTON, A. $\boldsymbol{e}$ t. al. Seleção de investimentos: aspectos e ferramentas relevantes na perspectiva dos gestores. In: ENCONTRO NACIONAL DE ENGENHARIA DE PRODUÇÃO, 24., 2004, Florianópolis. Anais... Florianópolis: ABEPRO, 2004, 1 CD-ROM.

BAKOKÉBAS JÚNIOR, B. et. al. Indicadores de segurança do trabalho para direcionamento do sistema de gestão de segurança e saúde no trabalho. In: ENCONTRO NACIONAL DE ENGENHARIA DE PRODUÇÃO, XXVI, 2006, Fortaleza. Anais... Fortaleza: ABEPRO, 2006, 1 CD-ROM.

BOS, E. H et al. The effects of occupational interventions on reduction of musculoskeletal symptoms in the nursing profession. Ergonomics. Jun 10; 49(7): 706-23, 2006.

CARRIVICK, P. J. et al. Evaluating the effectiveness of a participatory ergonomics approach in reducing the risk and severity of injuries from manual handling. Ergonomics. Jun 22; 48(8): 907-14, 2005.

CASAROTTO FILHO, N.; KOPITTKE, B. H. Análise de investimentos. 8. ed. São Paulo: Atlas, 1998.

FREITAS, S. C. Adaptação de um jogo de empresas para o ensino de análise de investimentos. 2002. 81 f. Dissertação (Mestrado em Engenharia da Produção) - Faculdade de Engenharia da Produção, Universidade Federal de Santa Catarina, Florianópolis.

GITMAN, L. J. Princípios de Administração Financeira. 7. ed. São Paulo: Harbra, 1997.

GUÉRIN, F. et. al. Compreender o trabalho para transformá-lo: A prática da ergonomia. São Paulo: Edgar Blucher, 2001.

HIRSCHFELD, H. Engenharia econômica. 3. ed. São Paulo: Atlas, 1986.

KASPCZAK, M. C. M.; SCANDELARI, L. Grau de utilização dos sistemas de fluxo de caixa na administração financeira das micro e pequenas empresas da cidade de Ponta Grossa. In: ENCONTRO NACIONAL DE ENGENHARIA DE PRODUÇÃO, XXVI, 2006, Fortaleza. Anais... Fortaleza: ABEPRO, 2006, 1 CD-ROM.

NUNES, E. L. S.; FRANZONI, A. M. B. Gestão do processo aliada a ergonomia para microempresas. In: ENCONTRO NACIONAL DE ENGENHARIA DE PRODUÇÃO, 24., 2004, Florianópolis. Anais... Florianópolis: ABEPRO, 2004, 1 CD-ROM.

POOSANTHANASARN, N. et. al. An ergonomics intervention program to prevent worker injuries in a metal autoparts factory. Southeast Asian Journal Tropical Medicine Public Health, Mar; 36 (2): 512-22, 2005.

SOUZA, A.; CLEMENTE, A. Decisões financeiras e análise de investimentos: fundamentos, técnicas e aplicações. São Paulo: Atlas, 1995.

STRAUSS, A.; CORBIN, J. Basics of qualitative research: grounded theory procedures and techniques. USA: SAGE, 1990.

WISNER, A. Questões epistemológicas em ergonomia e análise do trabalho. In: DANIELLOU, F. A ergonomia em busca de seus princípios: debates epistemológicos. São Paulo: Edgar Blücher, 2004, p. 29-55.

\section{Dados dos autores}

Nome completo: Thiago de Oliveira Pegatin

Filiação institucional: Faculdade de Medicina de São José do Rio Preto, FAMERP, Brasil

Departamento: Pós Graduação em Fisioterapia do Trabalho e Ergonomia

Função ou cargo ocupado: Professor de Pós-Graduação

Endereço completo para correspondência:

Rua: Pedro Copedê, 1130 Leste 
Jd. Modelo

17280-000- Pederneiras, SP- Brasil

Telefones para contato: (14) 91095420

e-mail: thiagopegatin@yahoo.com.br

Nome completo: Antonio Augusto de Paula Xavier

Filiação institucional: Universidade Tecnológica Federal do Paraná - UTFPR - Campus Ponta Grossa

Departamento: Pós Graduação

Função ou cargo ocupado: Professor de Graduação e de Pós Graduação

Endereço completo para correspondência:

Av. Monteiro Lobato, $\mathrm{km} 05$

Jardim Pitangui

84016-210 - Ponta Grossa, PR - Brasil

Telefones para contato: (42) 32204877

e-mail:augustox@utfpr.edu.br

Nome completo: Luiz Alberto Pilatti

Filiação institucional: Universidade Tecnológica Federal do Paraná - UTFPR - Campus Ponta Grossa

Departamento: Pós Graduação

Função ou cargo ocupado: Professor de Graduação e de Pós Graduação

Endereço completo para correspondência:

Av. Monteiro Lobato, $\mathrm{km} 05$

Jardim Pitangui

84016-210 - Ponta Grossa, PR - Brasil

Telefones para contato: (42) 32204805

e-mail:lapilatti@utfpr.edu.br

Nome completo: Ariel Orlei Michaloski

Filiação institucional: Universidade Tecnológica Federal do Paraná - UTFPR - Campus Ponta Grossa

Departamento: COALI - Coordenação de Alimentos

Função ou cargo ocupado: Professor de Graduação

Endereço completo para correspondência:

Av. Monteiro Lobato, km 05

Jardim Pitangui 
84016-210 - Ponta Grossa, PR - Brasil

Telefones para contato: (42) 32204800

e-mail:ariel@interponta.com.br 